\title{
Evaluation for Blunt Cerebrovascular Injury: Review of the Literature and a Cost-Effectiveness Analysis
}

\author{
(D) A. Malhotra, (D)X. Wu, (D) V.B. Kalra, (D). Schindler, (DC.C. Matouk, and (D)H.P. Forman
}

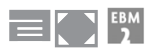

\begin{abstract}
BACKGROUND AND PURPOSE: Evaluation for blunt cerebrovascular injury has generated immense controversy with wide variations in recommendations regarding the need for evaluation and the optimal imaging technique. We review the literature and determine the most cost-effective strategy for evaluating blunt cerebrovascular injury in trauma patients.
\end{abstract}

MATERIALS AND METHODS: A comprehensive literature review was performed with data extracted to create a decision-tree analysis for 5 different strategies: anticoagulation for high-risk (based on the Denver screening criteria) patients, selective DSA or CTA (only high-risk patients), and DSA or CTA for all trauma patients. The economic evaluation was based on a health care payer perspective during a 1-year horizon. Statistical analyses were performed. The cost-effectiveness was compared through 2 main indicators: the incremental costeffectiveness ratio and net monetary benefit.

RESULTS: Selective anticoagulation in high-risk patients was shown to be the most cost-effective strategy, with the lowest cost and greatest effectiveness (an average cost of $\$ 21.08$ and average quality-adjusted life year of 0.7231 ). Selective CTA has comparable utility and only a slightly higher cost (an average cost of $\$ 48.84$ and average quality-adjusted life year of 0.7229 ). DSA, whether performed selectively or for all patients, was not optimal from both the cost and utility perspectives. Sensitivity analyses demonstrated these results to be robust for a wide range of parameter values.

CONCLUSIONS: Selective CTA in high-risk patients is the optimal and cost-effective imaging strategy. It remains the dominant strategy over DSA, even assuming a low CTA sensitivity and irrespective of the proportion of patients at high-risk and the incidence of blunt cerebrovascular injury in high-risk patients.

ABBREVIATIONS: $B C V I=$ blunt cerebrovascular injury; NMB = net monetary benefit; QALY = quality-adjusted life year

B lunt cerebrovascular injury (BCVI), defined as blunt trauma to the carotid and/or vertebral arteries, has a reported incidence ranging from $0.18 \%$ to $2.7 \%$ in blunt trauma admissions, higher in patients imaged with risk factors as defined by the Denver screening criteria and a high injury severity score. ${ }^{1,2}$ Arterial injury resulting in thrombosis and distal thromboembolism can result in BCVI-related stroke. A latent, asymptomatic period of

Received May 29, 2015; accepted after revision June 26.

From the Departments of Diagnostic Radiology (A.M., X.W., V.B.K., C.C.M., H.P.F.), Neurology (J.S.), and Neurosurgery (J.S., C.C.M.), Yale School of Medicine, New Haven, Connecticut.

Please address correspondence to Ajay Malhotra, MD, MMM, Department of Diagnostic Radiology, Yale School of Medicine, 333 Cedar St, New Haven, CT 065208042; e-mail: ajay.malhotra@yale

E Indicates article with supplemental on-line table and bibliography

Indicates article with supplemental on-line photos.

EBM Evidence-Based Medicine Level 2.

http://dx.doi.org/10.3174/ajnr.A4515
10-72 hours has been reported between injury and the onset of neurologic complications, during which antithrombotic therapy may improve neurologic outcome. Screening and early detection of BCVI within the clinically silent period can reduce the risk of stroke, improving the prognosis greatly. ${ }^{3-5}$ Multiple strategies have been suggested, generating debates between aggressive imaging for all blunt trauma admissions and selective screening of patients. ${ }^{6-8}$ In a recent study, $30 \%$ of patients had no radiographic or clinical risk factors, and the authors proposed more liberalized screening for BCVI. ${ }^{7}$

Digital subtraction angiography has been considered the reference standard for BCVI detection. ${ }^{9,10}$ Increasingly, CT angiography is used in emergency departments to assess patients. ${ }^{11}$ There is considerable variation in the reported sensitivity of CTA for BCVI detection in the literature, and its ability to replace DSA has not been validated, to our knowledge. In a multidisciplinary survey in 2011,60\% of practitioners in North America reported using CTA for screening, while $15 \%$ continue to use DSA. ${ }^{12}$ There 


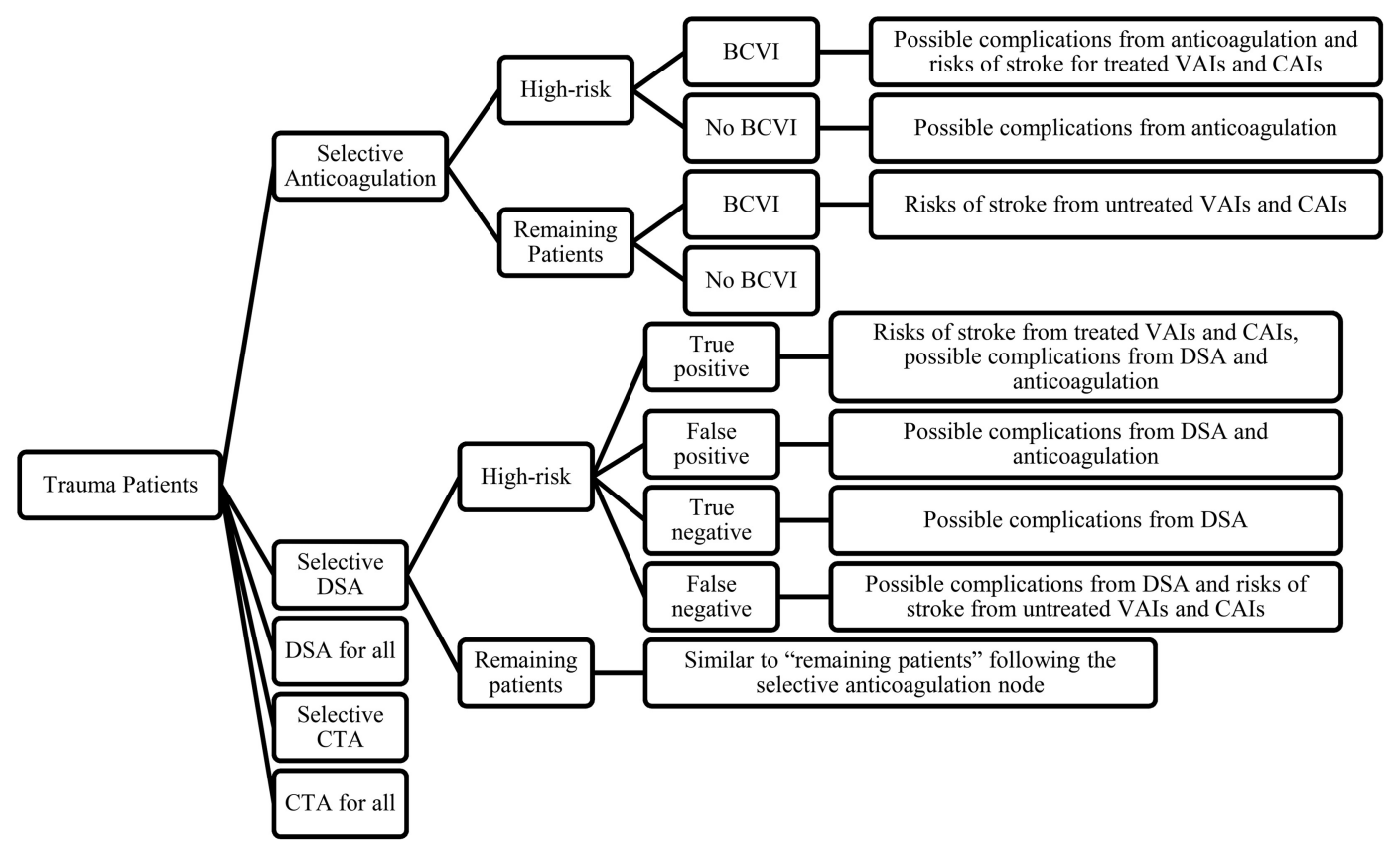

FIG 1. Flow chart of the simplified model. VAI indicates vertebral artery injury; CAl, carotid artery injury.

was wide variability in the choice of imaging and anticoagulation treatment of traumatic cerebrovascular injury, both on an individual basis and among specialties.

The Eastern Association for the Surgery of Trauma Management Guidelines in 2010 found no level 1 evidence for the appropriate technique for the screening and diagnosis of BCVI or for the treatment of BCVI. ${ }^{2}$

We decided to review the literature for the evaluation and management of BCVI and perform a cost-effectiveness study, incorporating all possible strategies and the direct costs as well as the complication rates of each strategy, in an attempt to determine the optimal strategy from a health care payer perspective.

\section{MATERIALS AND METHODS}

Approval from the institutional review board was not required because no actual patients were involved in this study, with all parameters obtained from literature.

\section{Model Description}

An economic decision analysis was developed to evaluate 5 possible strategies for adult blunt trauma admissions for BCVI (assuming an average age of 40 years) during a 1-year horizon. The mathematic model was constructed by using TreeAge Pro Suite 2014 (TreeAge Software, Williamstown, Massachusetts).

The 5 possible strategies were the following: no imaging with selective anticoagulation only for high-risk patients, DSA for all trauma admissions, DSA for high-risk patients (as per the Denver screening criteria), CTA for all admissions, and CTA for high-risk patients. For survivors of stroke, complications from anticoagulation and the angiographic procedure were discussed. Patients with false-positive results could also experience complications from anticoagulation and the angiographic procedure. A patient with true-negative findings would only experience complications from DSA or CTA, including a contrast-induced allergic-like reaction and contrast-induced nephropathy. Complications spe- cific to DSA included those at the arterial puncture site and from the catheter within the intracranial arteries, resulting in transient ischemic attack, stroke, and even death. As per the International Organization for Medical Physics, the risk of radiation-induced cancer and cancer death should not be estimated for doses of $\leq 100 \mathrm{mSv}$, markedly greater than the 3-6 mSv dose of radiation from CTA and the slightly higher dose from DSA. ${ }^{13-15}$ A patient with false-negative findings would have a higher risk of stroke due to a delayed diagnosis of BCVI. The survivors of stroke experience complications similar to those who have true-positive findings.

In selective screening strategies, the outcomes of patients in the high-risk population were discussed as those in DSA/CTA for all strategies, but with higher risks of stroke. Patients not in a high-risk population would not undergo an angiographic work-up and thus would not experience any procedural complications. Patients with BCVI within that population would have a delayed diagnosis with a higher risk of stroke.

In the selective anticoagulation strategies, high-risk patients would undergo anti-coagulation therapy, with associated costs and bleeding risks. The low-risk patients would not undergo any imaging or treatment with increased risk of stroke or a strokerelated mortality (Fig 1).

\section{Statistical Analysis}

The cost-effectiveness model was constructed from a health care payer perspective by using Medicare reimbursement values for costs when possible and literature values otherwise. This practice is recommended by the US Panel on Cost-Effectiveness in Health and Medicine. ${ }^{16}$ Outcomes were assessed as utility function, quantified by quality-adjusted life years (QALYs), based on World Health Organization cost-effectiveness guidelines. ${ }^{17}$

Two important indicators were used to assess the cost-effectiveness of the 5 strategies. The first was the incremental costeffectiveness ratio, defined as 


\begin{tabular}{ll}
\hline Signs and symptoms & Arterial hemorrhage/expanding cervical hematoma \\
& Cervical bruit \\
& Focal neurologic deficit \\
& Neurologic examination incongruous with head CT scan findings \\
& Stroke on secondary CT scan \\
& Maxillo facial fractures-Le Fort II or III fracture \\
& Cervical spine fracture patterns: extension into the transverse foramen, Cl-C3 vertebral fractures \\
Risk factors & Skull base fracture extending to involve the carotid canal \\
& Diffuse axonal injury with GCS score $<6$ \\
& Near hanging with anoxic brain injury \\
\hline
\end{tabular}

Note:- GCS indicates Glasgow Coma Scale. Table adapted from Cothren et al. ${ }^{34}$

Cost of Strategy $1-$ Cost of Reference Strategy

Expected Utility of Strategy 1 - Expected Utility of Reference Strategy.

The incremental cost-effectiveness ratio reflects the amount of additional expense for strategy 1 to achieve a full unit of incremental QALY with respect to the reference strategy, and the reference strategy is often chosen as the one of lowest cost. The incremental cost-effectiveness ratio was compared against the standard willingness-to-pay threshold of \$50,000/QALY. A strategy would be acceptable and cost-effective if it has the highest effectiveness among all strategies with an incremental cost-effectiveness ratio below willingness to pay.

The other indicator was net monetary benefit (NMB), defined as

$$
\text { Expected Utility } \times \text { Willingness to Pay }- \text { Cost. }
$$

The formula quantifies the benefits of a strategy in dollar amounts.

Base case calculation and a Monte Carlo simulation were performed. The base case calculation used the most probable value for each parameter, giving the average cost and effectiveness for a large cohort of patients. The Monte Carlo analysis with probabilistic sampling ran 10,000 iterations, simulating 10,000 hypothetic patients over the distributional range of each parameter. To test the robustness of our conclusion against different variables, we performed several sensitivity analyses. The sensitivity of CTA, the incidence of BCVI in all trauma admissions, the proportion of high-risk patients in all trauma admissions, the proportion of patients with BCVI having risk factors, and the risk of hemorrhage secondary to anticoagulation treatment varied from $0 \%$ to $100 \%$.

\section{Clinical Parameters}

The probability assigned to each path is the product of all probabilities along its nodes. These parameters were derived from multiple published studies. The incidence of BCVI in blunt trauma admissions was calculated as a weighted average of all studies included in the systematic review by Franz et $\mathrm{al}^{1}$ according to the number of patients in each study. The total number of blunt trauma admissions was 116,993 , and the total number of BCVIs was 1011 , giving an incidence of $0.86 \%$. Four studies reported the number of BCVIs with high-risk factors, and the weighted average was obtained to extrapolate the incidence of BCVI in high-risk patients. ${ }^{1,6-8,18}$ The proportion of high-risk populations in all blunt trauma admissions was derived from the weighted average of 2 studies totaling nearly 30,000 patients. ${ }^{6,7}$

\section{Costs and Outcomes}

Costs and utilities were assigned at each terminal node. Costs were quantified with US dollars in 2014 values, and utilities were measured in quality-adjusted life years. No discount rates were used because the study span was 1 year.

Costs were derived from Medicare when a single or several Current Procedural Terminology codes were involved and from published literature values otherwise, as recommended by the US Panel on Cost-Effectiveness Analysis in Health and Medicine. Specifically, the cost of stroke was calculated by dividing the lifetime stroke cost obtained by the difference between the average age at occurrence of BCVI (40 years of age) and the average life span of people in the United States (78.9 years of age). ${ }^{6,7,19-21}$ We acknowledge that our methodology for calculating the cost might be inaccurate, but the value is close to that reported in recent literature. $^{22}$

A list of all parameters and their values is presented on the On-line Table.

There are several assumptions made in this model:

1) The model was constructed with more than a 1-year horizon because BCVI is not a recurrent event. Minor complications with low costs and little reduction in QALY or those with rare incidence are excluded.

2) The major complications secondary to DSA include allergylike reactions (mild or severe), stroke (transient ischemic attack or permanent stroke), hematoma, and nephropathy.

3) CTA complications include allergy-like reactions (mild or severe) and nephropathy, with the same risks as those caused by DSA.

4) The major complication of anticoagulation treatment is hemorrhage.

5) Complications such as groin hematoma, contrast nephropathy, mild allergy-like reaction, and transient ischemic attack are assumed to incur costs but negligible reduction in effectiveness, with patients having a good outcome on recovery.

\section{RESULTS}

\section{Base Case Calculation}

In the base case calculation, parameters with distributions were assumed to be at its mean or most probable value. Expected costs and utilities for each strategy were calculated, and incremental cost-effectiveness ratios were compared against a willingness-to-pay threshold of \$50,000/QALY. The result showed that selective anticoagulation had the lowest cost and highest effectiveness (an average cost of \$21.08 and average QALY of 0.7231), and it would thus be the opti- 
mal strategy regardless of the willingness-to-pay threshold. Similarly, selective CTA would also be the optimal imaging strategy regardless of the value of the willingness-to-pay threshold (an average cost of \$48.84 and average QALY of 0.7229).

The summary is provided in Table 2 in the order of descending effectiveness.

\section{Monte Carlo Simulation}

A Monte Carlo simulation was performed with 10,000 iterations. The acceptability curve showed that selective CTA was the most cost-effective imaging strategy in all iterations, even at a willingness to pay of $\$ 100,000 / \mathrm{QALY}$.

A scatterplot of selective CTA against selective DSA is presented in Fig 2. The incremental effectiveness of selective DSA versus selective CTA in all iterations was negative (selective DSA has worse outcomes), and the incremental costs were positive (selective DSA is more expensive), suggesting that selective DSA is less cost-effective than selective CTA.

\section{Sensitivity Analysis}

The sensitivity of CTA, the incidence of BCVI in all trauma admissions, the proportion of high-risk patients in all trauma admissions, and the proportion of patients with BCVI having risk factors varied from $0 \%$ to $100 \%$. All 4 results showed selective anticoagulation to be the most cost-effective strategy and selective

Table 2: Base case calculation results

\begin{tabular}{lrc}
\hline \multicolumn{1}{c}{ Strategy } & Cost (\$) & Effectiveness (QALY) \\
\hline Selective anticoagulation & 21.08 & 0.7231074 \\
Selective CTA & 48.84 & 0.7229382 \\
Selective DSA & 284.62 & 0.7228022 \\
CTA for all & 419.11 & 0.7229337 \\
DSA for all & 5594.16 & 0.7196092 \\
\hline
\end{tabular}

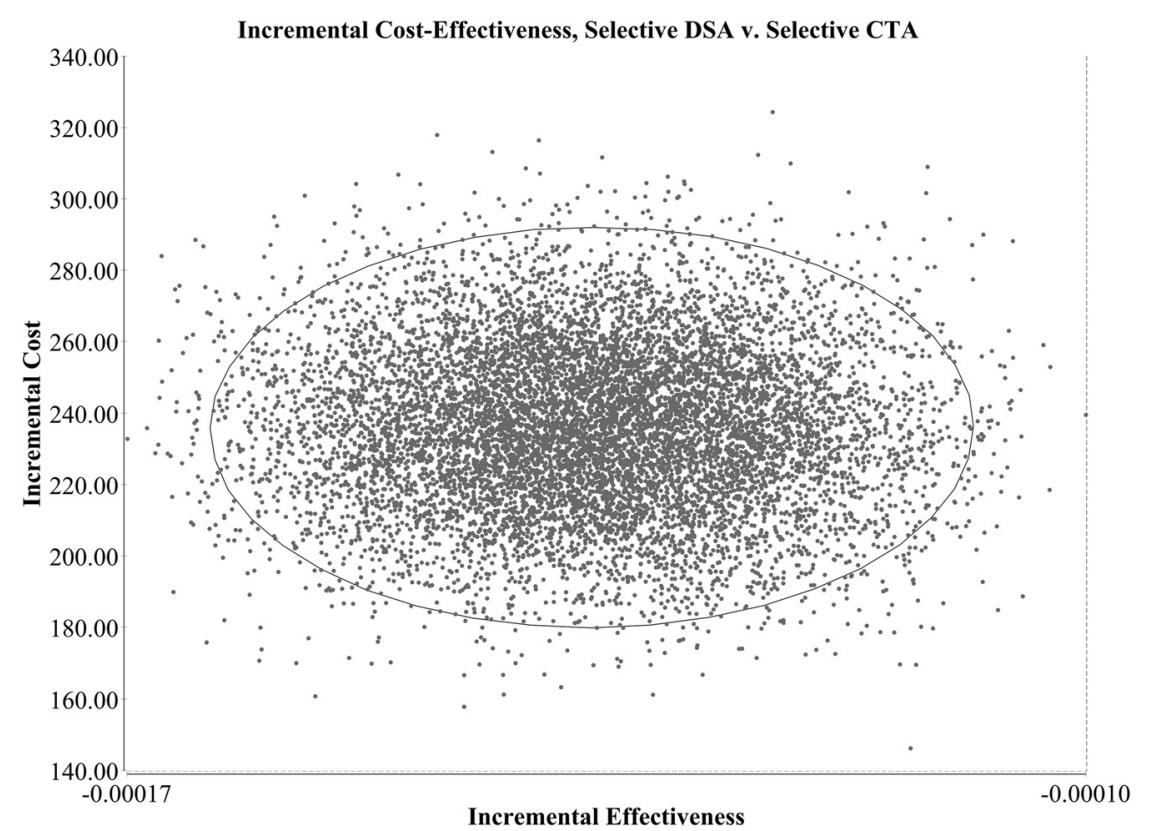

FIG 2. Scatterplot of incremental cost (cost of selective DSA and cost of selective CTA) versus incremental effectiveness (effectiveness of selective DSA and effectiveness of selective CTA). The incremental effectiveness in all iterations was negative (selective DSA has worse outcomes), and the incremental costs were positive (selective DSA is more expensive), suggesting that selective DSA is less cost-effective than selective CTA. and costs of DSA.
CTA to be the optimal imaging strategy (On-line Figs 1-4). As the proportion of high-risk patients increases, selective DSA progressively becomes an unfavorable strategy due to the complications

To compensate for the lack of literature reporting risk of hemorrhage secondary to anticoagulation specifically in trauma patients, we performed a sensitivity analysis, varying the incidence of hemorrhage. When the risk of hemorrhage secondary to anticoagulation is higher than $8 \%$, selective CTA is more cost-effective than selective anticoagulation and becomes the optimal strategy overall (On-line Fig 5).

\section{DISCUSSION}

Multiple studies have shown the benefits of early diagnosis and treatment of BCVI before onset of neurologic symptoms. Patients with BCVI can have significant morbidity and almost a $20 \%$ stroke-related mortality. ${ }^{3,4,20,23}$ Institution of adequate antithrombotic therapy was shown by Cothren et $\mathrm{al}^{20}$ in 2005 to reduce the incidence of ischemic neurologic events from $21 \%$ in untreated patients to $0.5 \%$ in treated patients. They found screening for BCVI with 4-vessel cerebrovascular angiography to be cost-effective when performed in patients at high-risk based on the Denver screening criteria. However, there has been significant controversy in defining both the patient population at risk for BCVI and the optimal screening technique.

In defining the high-risk population for BCVI, different protocols have been recommended, including the Denver screening criteria. ${ }^{4,20}$ However, by using the classically defined risk factors, Emmett et $\mathrm{al}^{24}$ found 16\% (19 of 117 patients) and Stein et $\mathrm{al}^{6}$ found $21.8 \%$ of BCVI patients with no risk factors. Using the Denver modification of screening criteria, Beliaev et $\mathrm{l}^{8}$ found the screening to have a sensitivity of $97 \%$ and a specificity of $42 \%$ and proposed that CTA be used only in high-risk patients.

The choice of technique should be based on comprehensive considerations, including both the sensitivity and specificity of the imaging technique as well as the risk of complications and costs. Increasing use of CTA has been reported to increase BCVI screening, reduce the time to diagnosis, and prevent stroke. $^{25,26}$ A negative CTA result has been shown to be associated with a low risk of subsequent neurologic complications in trauma patients. ${ }^{3,27,28}$ However DSA, the reference standard for BCVI detection, continues to be recommended despite being invasive, laborintensive, and available only in specialized centers. ${ }^{3,4,9,25,29-31}$ Most injuries missed on CTA are grade I injuries with luminal irregularity, but these have been shown to carry a significant risk of stroke. $^{3}$

In a meta-analysis comparing the accuracy of CTA against DSA for BCVI, Roberts et $\mathrm{al}^{10}$ reported a pooled sensitivity of $66 \%$ and specificity of $97 \%$ for 
CTA, with slightly better results for carotid-versus-vertebral artery injury. The sensitivity was significantly greater among studies using CT scanners with 16 versus $<16$ sections per rotation. However even with CT scanners with $\geq 16$ sections per rotation and CTA being read by neuroradiologists, the sensitivity remained below $80 \% .{ }^{10}$ Repeat injections during DSA can be performed if there are artifacts or suboptimal contrast filling, an option not available with CTA. In addition, DSA is performed by neuroangiographers with greater expertise in the assessment of BCVI, compared with CTA, which might be interpreted by general radiologists. A significant CTA learning curve has been described in which the diagnostic accuracy of CTA improved with time, with the specificity and negative predictive value climbing to $100 \%$ in the latter half of the study. ${ }^{29}$

There is great heterogeneity in the reported diagnostic performance of CTA and lack of uniform, unbiased comparison with DSA. Paulus et $\mathrm{al}^{11}$ reported an improved sensitivity of 64-detector row CTA and 32-detector row CTA, with a sensitivity of $68 \%$ and a specificity of $92 \%$ in 594 patients who met the screening criteria for BCVI and underwent both CTA and DSA. However, there was no blinding or mention of whether the findings could be seen on CTA retrospectively. All 52 false-negative CTA findings of BCVI were in 20 of 594 patients, and whether these CTAs were of optimal quality was not specified. The authors calculated that $0.4 \%$ of the study population could have been harmed if CTA had been used as the sole BCVI evaluation, while $0.5 \%$ of the patients had significant cerebrovascular complications from DSA. They, therefore, changed their protocol to perform DSA only in patients with positive CTA findings of BCVI or with unexplained neurologic deficits, despite the $68 \%$ sensitivity of CTA in their study. The justification given for DSA following positive CTA findings was the low specificity and positive predictive value of CTA $(92 \%$ and $36 \%$, respectively). However, these results are in stark contrast to the meta-analysis by Roberts et al in which CTA had a specificity of $99 \%$ for both carotid and vertebral artery injury, and DSA was proposed instead, with negative CTA results due to the low sensitivity of CTA.

Administration of antithrombotic agents in patients without contraindications has been shown to reduce the rate of neurologic sequelae after BCVI. ${ }^{4,23,32}$ No significant difference in outcome has been shown between heparin and antiplatelet agents. ${ }^{3,32,33}$ Although the reported bleeding complications with pelvic fractures or solid organ injuries are uncommon, there has been a hesitancy to use antithrombotic treatment. ${ }^{32}$

Given all these controversies, we constructed the model incorporating the factors discussed above when possible and performed sensitivity analyses otherwise. The results of our study demonstrate that despite assuming a relatively low sensitivity of CTA, selective CTA would still be more cost-effective due to its lower cost and low complication risks. The $66 \%$ sensitivity used in our model was from a meta-analysis totaling 5704 carotid and vertebral artery injuries. ${ }^{10}$ To compensate for the wide variability reported in the literature on the sensitivity of CTA versus DSA, we performed a sensitivity analysis by varying CTA sensitivities ranging from $0 \%$ to $100 \%$. The results showed that selective anticoagulation would be dominant among all 5 strategies. Among all imaging strategies, selective CTA had the highest NMB and would be the more optimal imaging test for patients with suspected BCVI because the complication rates of DSA were even higher than the incidence of BCVI in screened trauma admissions.

With different definitions of high-risk patients, ${ }^{15,17}$ the proportion of these patients among all trauma admissions and the incidence of BCVI in this group might also vary. Sensitivity analyses varying the proportion of high-risk patients among all trauma admissions and the incidence of BCVI among them were also performed to account for this limitation. When the values varied between $0 \%$ and $100 \%$, anticoagulation demonstrated the highest NMB, with selective CTA being the optimal imaging strategy under all possible scenarios.

In the base case calculation, selective anticoagulation of highrisk patients showed the highest cost-effectiveness. Antithrombotic treatment has been shown to reduce the stroke rate significantly in patients with BCVI. However, from an individual perspective, anticoagulating all patients may not be a feasible option, given the possibility of contraindications to anticoagulation and the risk of bleeding. In addition, the differences in both utility and cost between selective anticoagulation and selective CTA were relatively small, suggesting that selective CTA should be considered if an imaging study is to be performed to confirm or rule out the possibility of BCVI. Even when cost is not a major consideration, selective CTA results in higher utility among all imaging strategies.

Due to a lack of literature on the risk of bleeding after antithrombotic treatment in patients with blunt trauma, we performed a sensitivity analysis, which demonstrated that selective CTA replaced selective anticoagulation as the most cost-effective imaging strategy when the risk of hemorrhage secondary to anticoagulation was $>8 \%$.

Some of the studies included used the Memphis or other criteria to define patients at high-risk for BCVI. The heterogeneous use of screening criteria may lead to an inconsistent estimate of the BCVI incidence in high-risk patients.

Selective CTA in patients with risk factors according to Denver screening criteria would be the optimal and more cost-effective imaging strategy, even assuming a 100\% sensitivity of DSA and a low sensitivity for CTA.

\section{CONCLUSIONS}

Our analyses show that selective CTA in high-risk patients is the optimal and cost-effective imaging strategy for evaluation of BCVI in blunt trauma patients. Even if the CTA sensitivity is assumed to be low compared to DSA, or the proportion of patients at high-risk is over a wide range, selective CTA remains the preferred modality.

\section{REFERENCES}

1. Franz RW, Willette PA, Wood MJ, et al. A systematic review and meta-analysis of diagnostic screening criteria for blunt cerebrovascular injuries. J Am Coll Surg 2012;214:313-27 CrossRef Medline

2. Bromberg WJ, Collier BC, Diebel LN, et al. Blunt cerebrovascular injury practice management guidelines: the Eastern Association for the Surgery of Trauma. J Trauma 2010;68:471-77 Medline

3. Biffl WL, Moore EE, Elliott JP, et al. The devastating potential of blunt vertebral arterial injuries. Ann Surg 2000;231:672-81 CrossRef Medline 
4. Miller PR, Fabian TC, Croce MA, et al. Prospective screening for blunt cerebrovascular injuries: analysis of diagnostic modalities and outcomes. Ann Surg 2002;236:386-93; discussion 393-95 CrossRef Medline

5. Edwards NM, Fabian TC, Claridge JA, et al. Antithrombotic therapy and endovascular stents are effective treatment for blunt carotid injuries: results from longterm followup. J Am Coll Surg 2007;204: 1007-13; discussion 1014-15 CrossRef Medline

6. Stein DM, Boswell S, Sliker CW, et al. Blunt cerebrovascular injuries: does treatment always matter? J Trauma 2009;66:132-43; discussion 143-44 CrossRef Medline

7. Bruns BR, Tesoriero R, Kufera J, et al. Blunt cerebrovascular injury screening guidelines: what are we willing to miss? J Trauma Acute Care Surg 2014;76:691-95 CrossRef Medline

8. Beliaev AM, Barber PA, Marshall RJ, et al. Denver screening protocol for blunt cerebrovascular injury reduces the use of multi-detector computed tomography angiography. ANZ J Surg 2014;84:429-32 CrossRef Medline

9. Goodwin RB, Beery PR 2nd, Dorbish RJ, et al. Computed tomographic angiography versus conventional angiography for the diagnosis of blunt cerebrovascular injury in trauma patients. J Trauma 2009;67:1046-50 CrossRef Medline

10. Roberts DJ, Chaubey VP, Zygun DA, et al. Diagnostic accuracy of computed tomographic angiography for blunt cerebrovascular injury detection in trauma patients: a systematic review and metaanalysis. Ann Surg 2013;257:621-32 CrossRef Medline

11. Paulus EM, Fabian TC, Savage SA, et al. Blunt cerebrovascular injury screening with 64-channel multidetector computed tomography: more slices finally cut it. J Trauma Acute Care Surg 2014;76:279-83; discussion 284-75 CrossRef Medline

12. Harrigan MR, Weinberg JA, Peaks YS, et al. Management of blunt extracranial traumatic cerebrovascular injury: a multidisciplinary survey of current practice. World J Emerg Surg 2011;6:11 CrossRef Medline

13. Hendee W. Risk of medical imaging. Med Phys 2013;40:040401 CrossRef Medline

14. Sabarudin A, Yusof MZ, Mohamad M, et al. Radiation dose associated with cerebral CT angiography and CT perfusion: an experimental phantom study. Radiat Prot Dosimetry 2014;162:316-21 CrossRef Medline

15. Han A, Yoon DY, Kim ES, et al. Value of CT angiography for the detection of intracranial vascular lesions in patients with acute severe headache. Eur Radiol 2013;23:1443-49 CrossRef Medline

16. Weinstein MC, Siegel JE, Gold MR, et al. Recommendations of the Panel on Cost-Effectiveness in Health and Medicine. JAMA 1996; 276:1253-58 CrossRef Medline

17. Tan-Torres Edejer T, Baltusen R, Adam T, et al, eds. Making Choices in Health: WHO Guide to Cost-Effectiveness Analysis. Geneva: World Health Organization; 2003

18. Kopelman TR, Leeds S, Berardoni NE, et al. Incidence of blunt cerebrovascular injury in low-risk cervical spine fractures. Am J Surg 2011;202:684-88; discussion 688-89 CrossRef Medline
19. US Census Bureau. Expectation of Life at Birth, and Projections. 2008. http://www.census.gov/compendia/statab/2012/tables/12s0104.pdf. Accessed November 26, 2014

20. Cothren CC, Moore EE, Ray CE Jr, et al. Screening for blunt cerebrovascular injuries is cost-effective. Am J Surg 2005;190:845-49 Medline

21. Kaye D, Brasel KJ, Neideen T, et al. Screening for blunt cerebrovascular injuries is cost-effective. J Trauma 2011;70:1051-56; discussion 1056-57 CrossRef Medline

22. Hannon N, Daly L, Murphy S, et al. Acute hospital, community, and indirect costs of stroke associated with atrial fibrillation: population-based study. Stroke 2014;45:3670-74 CrossRef Medline

23. Fabian TC, Patton JH Jr, Croce MA, et al. Blunt carotid injury. Importance of early diagnosis and anticoagulant therapy. Ann Surg 1996;223:513-22; discussion 522-25 CrossRef Medline

24. Emmett KP, Fabian TC, DiCocco JM, et al. Improving the screening criteria for blunt cerebrovascular injury: the appropriate role for computed tomography angiography. J Trauma 2011;70:1058-63; discussion 1063-65 CrossRef Medline

25. Eastman AL, Muraliraj V, Sperry JL, et al. CTA-based screening reduces time to diagnosis and stroke rate in blunt cervical vascular injury. J Trauma 2009;67:551-56; discussion 555-56 CrossRef Medline

26. Schneidereit NP, Simons R, Nicolaou S, et al. Utility of screening for blunt vascular neck injuries with computed tomographic angiography. J Trauma 2006;60:209-15; discussion 215-16 CrossRef Medline

27. Berne JD, Cook A, Rowe SA, et al. A multivariate logistic regression analysis of risk factors for blunt cerebrovascular injury. $J$ Vasc Surg 2010;51:57-64 CrossRef Medline

28. Utter GH, Hollingworth W, Hallam DK, et al. Sixteen-slice CT angiography in patients with suspected blunt carotid and vertebral artery injuries. J Am Coll Surg 2006;203:838-48 CrossRef Medline

29. Malhotra AK, Camacho M, Ivatury RR, et al. Computed tomographic angiography for the diagnosis of blunt carotid/vertebral artery injury: a note of caution. Ann Surg 2007;246:632-42; discussion 642-43 CrossRef Medline

30. DiCocco JM, Emmett KP, Fabian TC, et al. Blunt cerebrovascular injury screening with 32-channel multidetector computed tomography: more slices still don't cut it. Ann Surg 2011;253:444-50 CrossRef Medline

31. Parks NA, Croce MA. Use of computed tomography in the emergency room to evaluate blunt cerebrovascular injury. Adv Surg 2012;46:205-17 CrossRef Medline

32. Cothren CC, Moore EE, Biffl WL, et al. Anticoagulation is the gold standard therapy for blunt carotid injuries to reduce stroke rate. Arch Surg 2004;139:540-45; discussion 545-56 CrossRef Medline

33. Wahl WL, Brandt MM, Thompson BG, et al. Antiplatelet therapy: an alternative to heparin for blunt carotid injury. $J$ Trauma 2002;52: 896-901 CrossRef Medline

34. Cothren CC, Biffl WL, Moore EE, et al. Treatment for blunt cerebrovascular injuries: equivalence of anticoagulation and antiplatelet agents. Arch Surg 2009;144:685-90 CrossRef Medline 